after all, it is only a graduation curve and it is open to anyone to find a better one! If Continental statisticians in the bulk do indeed hold the views of Mr. Buchanan-Wollaston, it can only be that they have not really studied and grasped the fundamental literature of the subject.

Biometrika Office,

Karl Pearson.

University College, London.

${ }^{1}$ NATURE$_{136}, 182$, August 3, 1935.

\section{Blood Group Inheritance}

Several theories of the inheritance of human blood groups have been proposed, but none has been completely satisfactory.

(i) The theory of two independent pairs of Mendelian factors, proposed by Hirschfeld and von Dungern $^{1}$, leads to the equilibrium condition

$$
p s=q r
$$

where $p, q, r, s$ are the frequencies of the groups $A B, A, B, O$. But ${ }^{2,5}$ in all observations of the distribution of the groups,

$$
p s<q r .
$$

(ii) The corresponding condition arising from Bernstein's multiple allelomorph theory ${ }^{3,5}$ is

$$
\sqrt{q+s}+\sqrt{r+s}=\sqrt{s}+\sqrt{p+q+r+s} .
$$

This is satisfied with sufficient accuracy in all observed distributions, but this theory fails to account for the observed occurrence of a small percentage of groups $A B$ and $O$ in the offspring of $A B \times O$ matings.

(iii) Bauer ${ }^{4}$ proposed to account for this by the mechanism of 'crossing-over'. His theory is the subject of criticisms in a paper now in course of preparation, in which we shall show that the following theory, which is not exactly what Bauer stated, is the one to which his premises should have led:

(iv) The blood groups are inherited in association with two linked pairs of Mendelian factors, $A, a, B, b$. There are thus four kinds of gametes,

$$
A B, A b, a B, a b \text {; }
$$

and ten genotypes :

$A B . A B, A B . A b, A B . a B, A B . a b, A b . a B$-phenotypically group $A B$;

$A b . A b, A b . a b-\operatorname{group} A$;

$a B . a B, a B . a b$-group $B$

$a b . a b$-group $O$.

If crossing-over takes place, we may suppose that there is a certain probability $\omega$ that the genotype $A B . a b$ transmits the gametes $A b$ and $a B$ normally transmitted by the genotype $A b . a B$; and the same probability of vice versa.

In Bauer's theory (iii), $\omega$ is about 0.055 . If $\omega=0$ and the first four genotypes in the above scheme are initially (and therefore permanently) absent from the population, the theory (iv) reduces to (ii). With $\omega=\frac{1}{2}$, (iv) is indistinguishable from (i).

The theory we actually propose is (iv) with $\omega=0$. (This also gives results indistinguishable from (i) in all matings which do not involve the distinction between the genotypes $A B . a b$ and $A b \cdot a B$.) It is unnecessary to postulate crossing-over, since with this theory $A B \times O$ matings would anyhow give rise to some $A B$ and $O$ offspring. But, further, crossingover is definitely precluded, since it may be shown to lead to the same equilibrium condition as the theory (i). There is no corresponding condition when $\omega=0$; that is to say, any distribution of the groups can be in stable equilibrium provided that the genotypes are suitably distributed. But for equilibrium distributions in which $A B$ is rare compared with the other groups, the theory approximately coincides with (ii); and therefore, as in that theory,

$$
\sqrt{q+s}+\sqrt{r+s}=\sqrt{s}+\sqrt{p+q+r+s}
$$

approximately. This, as already pointed out, agrees with observation, and therefore (since $p$ is generally about 5 per cent) corroborates the new theory.

The rare recorded occurrences ${ }^{5}$ of $A$ offspring from $O \times O$ matings, of $A B$ and $B$ from $A \times O$, and of $A B$ and $A$ from $B \times O$, cannot be accounted for genetically by any of these theories. They must still be put down to mistaken parentage or faulty technique in group determination.

Thus the theory proposed has all the advantages of its predecessors, with fewer disadvantages.

J. Froulixes Edwards.

University College Hospital, London.

Department of Mathematics,

\section{M. H. Etherington.} The University, Edinburgh. July 6 .

${ }^{1}$ Hirschfeld and von Dungern, Z. Immun. Exp. Therapie, 6, 284 1910 .

"Lattes, "The Individuality of the Blood". O.U.P. (1932)

- Bernstein, Z. Ind. Abst. u. Vererb., 37, 237; 1925.

Furuhata, Japan. Medical World, i, No. 7, 1927.

\section{Technique of Factor Analysis}

FActor analysis is a subject upon which Prof. G. H. 'Thomson, Dr. Wm. Brown and others have frequently written letters to NATURE. This analysis is concerned with a selected population of $n$ individuals each of whom has been measured in $m$ tests. The (m) $(m-1) / 2$ intercorrelations for these $m$ variables are subjected to either a Spearman or other factor analysis.

The technique, however, can also be inverted. We begin with a population of $n$ different tests (or essays, pictures, traits or other measurable material), each of which is measured or scaled by $m$ individuals. The $(m)(m-1) / 2$ intercorrelations are then factorised in the usual way.

This inversion has interesting practical applications. It brings the factor technique from group and field work into the laboratory, and reaches into spheres of work hitherto untouched or not amenable to factorisation. It is especially valuable in experimental aesthetics and in educational psychology, no less than in pure psychology.

It allows a completely new series of studies to be made on the Spearman 'central intellective factor' $(g)$, and also allows tests to be made of the Two Factor Theorem under greatly improved experimental conditions. Data on these and other points are to be published in due course in the British Journal of Psychology.

Psychological Laboratory,

W. Stephenson.

University College,

Gower Street,

London, W.C.1. June 28. 